\title{
From a syndrome to a social condition: providing palliative care to people with advanced dementia
}

Wilson Correia de Abreu ${ }^{1}$

Dementia is one of the biggest public health challenges in societies; over 35 million people worldwide live with the condition. This number is expected to triple by 2050. Substantial increases in health and social care costs are required to deal with this major public health problem. Thus, this explain why dementia has become a globally alarming issue.

Dementia is a syndrome of diverse aetiology, characterised by progressive cognitive decline, functional impairment and dependence in self-care. Usually, the syndrome is associated with older people, and it is often accompanied by co-morbidities, pain and different organ dysfunctions. Studies show the bewilderment, distress and exhaustion relatives and loved ones feel when caring of people with dementia.

The progression of dementia is often separated into mild, moderate and severe, corresponding to early, mid and late or advanced stages. Much has been done worldwide to support people in the early stages of dementia - usually, most people function independently. The middle to late stages of the disease often signals a loss of autonomy, independence and reduction in physical and cognitive function. In this advanced stage, there is frequently a substantial or total dependence in self-care and need of palliative care ("extended palliative care"); relevant text about the extended palliative phase of dementia often included descriptions of symptoms, prognosis and the difficulties in recognising the phase itself ${ }^{(1)}$.

The onset of advanced dementia, as a life-limiting and life-threatening syndrome, generates a great concern for all those involved. Even the increasing support being provided to patients with dementia, pain is often underrated and there are frequently high levels of suffering. Other neuropsychiatric symptoms like depression are also commonly associated with dementia; persons are more exposed to complications, recurrent hospitalization and questionable invasive therapies. In many cases, death is caused by other pathologies such as pneumonia, dehydration and failure of vital organs. Palliative care can contribute to reduce hospitalization, healthcare expenditure and provides comfort and well-being for patients and families.

Palliative Care means delivery of care to patients suffering from severe and/or advanced and progressive illnesses; palliative care provides relief from pain and other signals and symptoms experienced by people with a strong dependence on self-care, aiming to promote quality of life and well-being. Recently, in the European Project Palliare, a Best Practice Statement was made to support palliative care to people with advanced dementia. It includes the identification of good practices targeted at people with advanced dementia, who are not yet requiring end-of-life care.

Suggested that the disease trajectories of people in need of palliative care are very different and require distinctive responses. The authors provide evidence-based recommendations. I would like to emphasise four, given its important for the conception of palliative care for people with advanced dementia: (1) Palliative care should provide best possible quality of life and promote comfort, enabling to identify needs and manage signs

${ }^{1}$ Escola Superior de Enfermagem do Porto. PhD, Professor Titular. Porto, Portugal.

Corresponding author: Wilson Correia de Abreu

Rua Dr. António Bernardino de Almeida, 4200-072 - Porto, Portugal. E-mail: wjabreu@esenf.pt 
and symptoms; (2) Addressing the patient's spiritual need, wishes, beliefs, personal values and support this person in decision-making, involving the multidisciplinary team will highly contribute to dignify the support provided, humanize care and uphold respect for the person with dementia and family; (3) To preserve the continuity of care, even in case of hospitalization; (4) Palliative care has also as target the family: family has to be ready to deal with the progression of the syndrome and the proximity of death, in order to be prepared to deal with mourning and to make adjustments after death occurs ${ }^{(2)}$.

Nurses play an irreplaceable role in this phase (management of signals and symptoms, pain control, provision of basic care, comfort). It is also imperative that they are prepared for autonomous non-invasive and non-medication interventions that stimulate senses and provide comfort. The spiritual comfort is also relevant because it interferes not only with beliefs but also with the attitude towards death and mourning. Furthermore, nurses and the multidisciplinary team roles' have to include the design and validation of instruments to assess functionality, prognosis, patients' needs and to support clinical decision-making.

The upcoming creation of the European Academy in Dementia, as part of the European project Palliare, will be a way to promote networks of researchers and practitioners worldwide who are interested in dementia: we all understand how important it is that the person with dementia and their family remain as the main focus of care, research and education.

\section{References}

1. Hanson E, Hellstrom A, Sandvide A, Jackson G, MacRae R, Waugh A, et al. The extended palliative phase of dementia - An integrative literature review. Dementia [Internet]. 2016 [cited 2017 Jan 5];1-27. Available from: http://journals.sagepub.com/doi/pdf/10.1177/1471301216659797

2. Van der Steen J, Radbruch L, Hertogh C, Boer M, Hughes J, Larkin P, et al. White paper defining optimal palliative care in older people with dementia: a Delphi study and recommendations from the European Association for Palliative Care. Palliative Med. 2014; 28(3):197-209. 